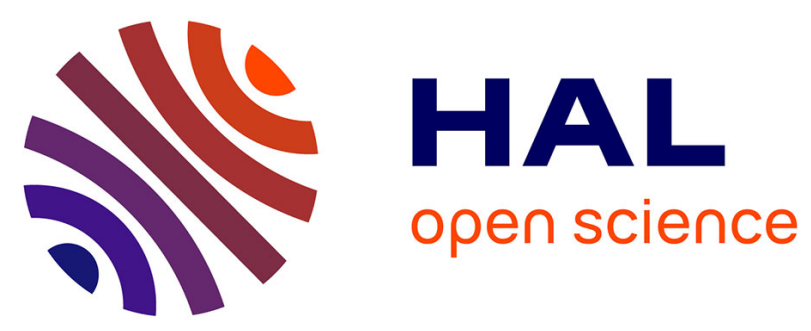

\title{
Laser-induced densification of fused silica using spatially overlapping sub-30 fs pulses
}

\author{
Ben Mcmillen, Olivier Uteza, Raphaël Clady, Nicolas Sanner, Marc Sentis, \\ Farhang Nabiei, Ya Cheng, Yves Bellouard
}

\section{- To cite this version:}

Ben Mcmillen, Olivier Uteza, Raphaël Clady, Nicolas Sanner, Marc Sentis, et al.. Laser-induced densification of fused silica using spatially overlapping sub-30 fs pulses. Journal of Applied Physics, 2020, 128 (8), pp.083107. 10.1063/5.0011317 . hal-02982215

\section{HAL Id: hal-02982215 \\ https://hal-amu.archives-ouvertes.fr/hal-02982215}

Submitted on 28 Oct 2020

HAL is a multi-disciplinary open access archive for the deposit and dissemination of scientific research documents, whether they are published or not. The documents may come from teaching and research institutions in France or abroad, or from public or private research centers.
L'archive ouverte pluridisciplinaire HAL, est destinée au dépôt et à la diffusion de documents scientifiques de niveau recherche, publiés ou non, émanant des établissements d'enseignement et de recherche français ou étrangers, des laboratoires publics ou privés. 


\title{
Laser-induced densification of fused silica using spatially overlapping sub- 30 fs pulses
}

\author{
Ben McMillen ${ }^{1, a}$, Oliver Uteza², Raphaël Clady², Nicolas Sanner², Marc Sentis², \\ Farhang Nabiei ${ }^{3}$, Ya Cheng ${ }^{4}$, and Yves Bellouard ${ }^{1}$ \\ ${ }^{1}$ Galatea Lab, STI/IMT, École Polytechnique Fédérale de Lausanne, Rue de La Maladière 71b, CH-2002 Neuchâtel, \\ Switzerland \\ ${ }^{2}$ Aix-Marseille University, CNRS, LP3 UMR 7341, 13288 Marseille, France \\ ${ }^{3}$ Electron Spectroscopy and Microscopy Lab, STI/IMT, École Polytechnique Fédérale de Lausanne, Section 3, CH-1015 \\ Lausanne, Switzerland \\ ${ }^{4}$ Shanghai Institute of Optics and Fine Mechanics, 899 Huiwang E Rd, Jiading Qu, Shanghai Shi, 201800, China
}

Over the past several years, non-ablative femtosecond laser exposure with spatially overlapping (i.e. spatially cumulative) pulses has emerged as a key process in three-dimensional writing of patterns in bulk of dielectric substrates. When temporally non-cumulative and combined with post-processing steps, this process defines a novel manufacturing technique in fused silica, finding use in a broad number of applications, including - but not limited to - micromechanics, integrated optics, microelectronics, microfluidics, information storage, and combinations of these fields for novel integrated sensing applications. For fused silica, evidence has shown that there is a pulse-length duration threshold around $200 f s$, marking the boundary between two radically different characteristic material modification regimes, each leading to a specific application. Pulsewidths below $200 f s$ lead to localized densification, enabling the direct-write of optical waveguides, while pulse-widths above this value produce self-organized nanostructures causing a localized volume expansion and enhanced etching susceptibility to various chemicals. Here, we focus our attention on the regime below $200 f_{s}$, using low repetition rate, temporally noncumulative pulses. In particular, we use very short pulses, i.e. in the range of $30 f s-$ a regime as yet unexplored from the viewpoint of spatially cumulative modifications. Our goal is to understand how structural modifications obtained by overlapping pulses evolve with varying pulse overlap, and how shorter pulse duration may correlate with higher material densification. This knowledge is particularly important for the next generation of photonics devices, where increasing the level of laser-induced densification is a key factor for high-density photonic integration.

\section{INTRODUCTION}

The use of shorter pulse-widths has many advantages for laser materials processing. As the pulse-width is reduced, higher intensities can be accessed in the focal region, even in the case of intensity clamping, which scales with respect to the inverse of the pulse-width as $\left(1 / t_{p}\right)^{1 /(K-1)}$, where $t_{p}$ is the pulse-width and $K$ is the number of photons involved in the process of multiphoton ionization. This holds even if the scaling is weak in large bandgap (high K) dielectric materials [1]. Higher intensity (shorter pulses) leads to higher localized energy (higher free electron density) and no time for any energy upload loss, all of which are favorable conditions to reach locally higher pressure transients, and thus stronger material densification.

a) Author to whom correspondence should be addressed. Electronic mail: ben@ mcmillen.eu 
However, the main difficulty when shortening the pulse-width is that higher peak powers also trigger strong nonlinear effects [2] which can be detrimental for achieving controlled and reproducible modifications. This fact is particularly evident when the beam must be focused tightly. Effects such as self-focusing, filamentation, and pulse break-up all contribute to material modifications, which at first due to their unstable nature, may appear unsuitable for applications in bulk material processing and internal structuring, such as waveguide fabrication [3]. Indeed, since the seminal paper from Marburger [4], which lays out the groundwork for self-focusing and filamentation, the so-called critical power for self-focusing [2,4,5] has often been a discouraging factor for shortening pulses because of the possible instability and collapse of the laser focus. While past studies (see for instance [6,7]) have dealt with the use of lasers with pulse-widths comparable to the values used here, our work covers a regime of varying spatial overlap between pulses, not previously explored. Here the term 'spatial overlap' means the overlap between resulting material modifications left behind in the substrate. For a fixed repetition rate (here $100 \mathrm{~Hz})$, the amount of spatial overlap between laser-affected zones varies as a function of the translation speed with respect to a fixed focusing objective. The low repetition rate of $100 \mathrm{~Hz}$ allows easy transition from 'single pulse' to 'many pulse' (i.e. 'spatially overlapping') exposure regimes, while keeping the translation speed of the substrate reasonable. Interestingly, in order to achieve the necessary fluence required for material modification given our focusing conditions and repetition rate, we must work with pulse energies well above the critical power for self-focusing, as defined in [5].

In this work, we explore the effects of these laser pulses in terms of structural and density changes induced in the material within the laser-affected zone (LAZ). Having in mind laser direct-write processes, we specifically explore the pulse energy versus pulse overlap space. Our findings indicate that the rate of energy deposition is highly influential on the outcome of the machining process: identical cumulative energy doses (i.e. more pulses at low energy versus fewer pulses at high energy) do not necessarily yield the same outcome. In general, lower pulse energy is found to be more beneficial for producing continuous modifications, which in turn yield a highly densified material. A further increase of the pulse energy results in the formation of a wide variety of microstructural transformations as a result of stronger nonlinear interactions, ultimately serving as the liming factor in measuring higher material density using our chosen measurement technique.

\section{EXPERIMENTAL SETUP}

\section{A. Laser setup and exposure conditions}

Laser pulses were supplied by the ASUR laser system at the laboratory LP3 in Marseille [8], producing $800 \mathrm{~nm}$ pulses at a repetition rate of $100 \mathrm{~Hz}$, and a duration of $\sim 27 f s$ for the beam line used in this experiment. To limit pulse dispersion, a $90^{\circ}$ off-axis parabola with a focal length of $15 \mathrm{~mm}$ was chosen for beam delivery to the sample, resulting in an equivalent NA of 0.25 for an input beam diameter of $\sim 8 \mathrm{~mm}$ and a measured spot size of $3 \mu \mathrm{m}$. Glass substrates were positioned relative to the 
laser focus using a combination of manual and piezo-driven flexure stages, providing both long and short-range motion respectively.

Exposure conditions were determined using an equation based on the parameter known as deposited energy density [9], which gives a measure of the fractional laser fluence passing through the modified region of the glass, while concatenating several parameters of the machining process, as follows:

$$
\mathrm{E}_{\mathrm{d}}=\underbrace{\frac{4 \mathrm{E}_{\mathrm{p}}}{\pi\left(2 \mathrm{w}_{0}\right)}\left(\frac{\mathrm{f}}{\mathrm{v}}\right)}_{\text {Total fluence }} \underbrace{\left(\frac{\mathrm{w}_{\text {laz }}}{2 \mathrm{w}_{0}}\right)}_{\text {LAZ ratio }},
$$

where $E_{p}$ is the pulse energy, $2 w_{o}$ and $w_{\text {laz }}$ are the width of the focused spot and laser-affected zone (LAZ) respectively, $f$ is the repetition rate of the laser, and $v$ is the scanning velocity. This incoming fluence (or 'deposited energy') is useful for comparing exposure conditions as it concatenates several parameters of the machining process. The original definition [9] only considers the width of the LAZ with two basic assumptions: that this is comparable to the width of the focal spot and that it remains constant over a wide range of exposure conditions. These assumptions are valid when considering exposures at high repetition rates (several hundred kilohertz), where the width of the LAZ is relatively invariant as the result of exposure to many thousands of pulses. In this experiment however, the width of the LAZ varies greatly due to contributions from nonlinear effects, ranging from a few hundred nanometers to several microns. These differences in exposure regimes are taken into consideration by including the additional term $w_{l a z} / 2 w_{o}$, which takes the fractional area of the total fluence passing through the LAZ. Note that this parameter only gives an indication of how much energy passes through the modified region, but does not account for the exact amount of energy effectively 'absorbed' by the material, which unfortunately could not be easily measured.

The exposure range was determined using known limitations of the machining system as defined by the speed of the stages and the repetition rate of the laser. Using the measured focal waist as a metric, the maximum exposure was computed from the minimum velocity of the piezo stages giving approximately 75 pulses per focal waist at a speed of $4 \mu \mathrm{m} / \mathrm{s}$ and $100 \mathrm{~Hz}$, while the minimum was defined as the speed at which individual pulses no longer overlap, or $300 \mu \mathrm{m} / \mathrm{s}$. Pulse energy was chosen in a similar fashion, setting the lower bound at $0.25 \mu \mathrm{J}$, i.e. just above the threshold for modification as determined through exploratory experiments based on the criteria of being observable under a cross-polarized microscope, and the maximum at 3 $\mu \mathrm{J}$, which was chosen in an effort to limit catastrophic substrate damage at high deposition rates. These parameters, combined with the measured width of the LAZ, give a range of deposited energy of $\sim 0.001$ to $24 \mathrm{~J} / \mathrm{mm}^{2}$. Note that for the deposited energy calculations defined by Equation 1, the width of the LAZ was not known prior to these experiments, and was determined from cross-sectional analysis of the modified region using scanning-electron microscope (SEM) imaging. 


\section{B. Sample preparation}

To study the effects of laser-induced volume change we employ a method based on the deflection of laser-machined glass micro-cantilevers, which enables precise measurement of volume changes in the laser affected zones [10], and can be accurately correlated with residual stress measurements using photoelasticity [11,12]. The cantilevers were machined in several $25 \times 25 \times 0.25 \mathrm{~mm}$ fused silica substrates using a laser-assisted wet-etch process [13,14]. Each substrate contained a total of 26 cantilevers, arranged in an $\sim 8 \times 8 \mathrm{~mm}$ linear array at the center. The design of an individual cantilever, which is shown in Fig. 1(a), consists of a $200 \mu \mathrm{m}$ wide by $7500 \mu \mathrm{m}$ long glass beam, anchored to the substrate at one end. The free end was machined with a set of opposing matched points, allowing measurement of both in- and out-of-plane deflection. Due to the low repetition rate of the laser used, the substrate thickness, cantilever dimensions, and size of the exposure region were chosen to provide the best compromise between exposure time and measurement sensitivity.

FIG. 1. (a) cantilever device design for a single, monolithic glass beam, (b) definition of parameters used in calculating stress and strain from a given measured deflection, (c) experimental setup depicting the focusing arrangement and additional parameters used in the stress calculations. See section IID for a description of the mechanical modeling methods used for estimation of the strain within the LAZ based on deflection measurements.

In order to provide easier access to the LAZ for imaging and analysis, an additional sample was machined. Using the same exposure conditions as for the cantilevers, sets of 5 lines were written perpendicular to the edge of the sample, for both perpendicular and parallel polarization (with respect to the writing direction), with a spacing of $10 \mu \mathrm{m}$ for each exposure and $100 \mu \mathrm{m}$ between exposure group. This sample was then cut and polished in to reveal a cross-sectional view of the laser-modified region in preparation for imaging and Raman analysis. Finally, the microstructure within the LAZ was imaged in an SEM after a brief etch in $2.5 \%$ hydrofluoric acid $[13,15]$.

\section{Measurement Techniques}

\section{Deflection measurements}

Fig. 1(b) outlines the basic method for measuring laser-induced volume change. In this technique, a modified layer is created at the base of a suspended glass cantilever by scanning a focused femtosecond laser just below the surface. This layered arrangement of bulk and modified glass creates a composite-like structure, which responds by bending in relation to volumetric changes induced within the laser-modified region. As this bending is typically very small (considering volume changes of less than a few percent), the additional length of the cantilever provides mechanical amplification, 
converting the small bending moment induced by the composite structure into a measurable mechanical deflection at the tip.

Only a short segment of each cantilever was exposed (no more than $500 \mu \mathrm{m}$ ) by writing evenly-spaced lines parallel to the long dimension of the cantilever, with line spacing varying between 3 and $5 \mu \mathrm{m}$ depending on the exposure conditions used. During the writing step, the laser-induced modifications were kept close to the top surface of the cantilever, but still contained within the bulk glass (Fig. 1(c)). This spatial localization of the modifications provides the maximum possible deflection while mitigating stress release through defect-induced crack formation at the surface.

Cantilever deflection was measured with a white-light interferometer (Wyko NT-1100), using the surrounding cantilever frame as a reference surface for flatness and tilt correction. Sample pre-strain was accounted for through inclusion of offset measurements of un-machined cantilevers in each substrate.

\section{2. $\mu$-Raman imaging}

2D Micro-Raman imaging was used to investigate the structural changes induced by short-pulse laser exposure at the molecular scale. For this measurement, features written with a pulse energy of $0.75 \mu \mathrm{J}$ were selected, as this exposure produced deflection results with the most consistency. Using the secondary lines sample, the LAZ of each exposure condition was mapped using a 50x objective over an area of 10 x $50 \mu \mathrm{m}$ and a $2.5 \mu \mathrm{m}$ step size. At an excitation wavelength of $488 \mathrm{~nm}$, two averaged measurements with an integration time of 30s were taken for each point over the spectral range of $200-1000 \mathrm{~cm}^{-1}$. After measurement, the $\omega_{3}$ peak was used for normalization, providing a baseline for measuring the position, intensity, and width (at $70 \%$ of the peak) of the main band $\left(\omega_{1}\right)$, as well as the position and intensity of the $D_{l}$ and $D_{2}$ peaks [16-26].

\section{Birefringence imaging}

Additional information about the distribution of stress around the LAZ was obtained through birefringence imaging. For these measurements, we only concentrate on the absolute magnitude of the retardance [12,27] by imaging each LAZ with a standard microscope, equipped with a liquid-crystal compensator (CRi Instruments, VariLC) and controlled by OpenPolScope $[28,29]$ The resulting grey scale images were then cropped and normalized to the maximum calibrated retardance of $10 \mathrm{~nm}$. Using a peak-finding algorithm written in Matlab, prominent peaks were selected along a perpendicular line through the highest intensity retardance found in unmodified material between the lines. A Gaussian curve was then fit to each set of points, with additional anchor points near the ends of the trace to improve the fit, giving an approximation of the overall average strain between the lines. 
Here the strain was computed from the measured retardance, first by computing the difference of principal stress using the stress-optic law, followed by a conversion to strain using stress-strain relationships and the Young's modulus for silica glass.

\section{Thin sample preparation and TEM studies}

To further investigate the presence of nanostructures within the laser-modified regions of each sample, thin sections were prepared for analysis via transmission electron microscopy (TEM) as follows. Each bulk sample was introduced into a ZEISS NVision 40 dual beam (electron and $\mathrm{Ga}^{+}$ion beam) instrument. The cross-sections of the laser-modified regions were analyzed by electron beam imaging using secondary and back-scattered electron emission to select specific sites for thin section preparation using the focused ion beam (FIB) in-situ lift-out technique [30]. Each region of interest was coated with a $\sim 2$ um thick carbon deposition induced by ion beam exposure. The sample was then milled with $30 \mathrm{kV}$ and beam currents ranging from $27 \mathrm{nA}$ down to $700 \mathrm{pA}$ to obtain a slice of $\sim 1 \mu \mathrm{m}$ thickness. This slice was then cut out and transferred to a copper grid, followed by thinning to obtain the required thickness of $100 \mathrm{~nm}$ for TEM analysis. To avoid substrate damage, low beam currents ranging from 80 to 700 pA were used. Finally, the thin section was polished using an ion beam exposure of $2 \mathrm{kV}$ and $25 \mathrm{pA}$. This method was used to prepare two thin sections (TS1 and TS2) from different regions within the cross-sections of the laser-modified lines, as shown in Fig. 2 below.

FIG. 2. SEM images of modified regions corresponding to thin sections TS1 and TS2, with exposure parameters of $3 \mu \mathrm{J} / 16 \mu \mathrm{m} / \mathrm{s}$ (TS1) and $3 \mu \mathrm{J}, 4 \mu \mathrm{m} / \mathrm{s}$ (TS2). All modifications shown in (a) and (b) were written with parallel polarization.

\section{Calculation of strain from measured deflection}

The mechanical behavior of the laser-exposed cantilevers is modeled as a composite stack of three materials [31-34], consisting of the laser-exposed region sandwiched in between two layers of pristine, un-modified glass. As deflections are small, we then consider a modified form of Euler-Bernoulli beam theory [31,34-36]. The approach is as follows: we assume that the strain at the ends of and throughout the composite structure must be the same, and that only the stress in each layer will be different. We additionally assume, for the sake of simplicity, that the LAZ is a homogeneously modified region. Given that the deflection at the cantilever tip is known (measured), the curvature of the modified composite can then be determined using simple trigonometry, and the strain calculated using the following equation (see Fig. 3 for reference): 


$$
\varepsilon_{\mathrm{avg}}(\mathrm{y}) \approx \mathrm{y} \frac{\delta}{\mathrm{SL}}
$$

where $\varepsilon(y)$ is the height-dependent strain along the thickness of the cantilever, $y$ is the distance from the neutral line, $\delta$ is the cantilever deflection, $S$ is the laser-exposed length, and $L$ is the un-exposed length of the cantilever. The full term $\delta / S L$ gives the curvature of the beam, taking into account the added cantilever length for amplification. Note that the value taken for $y$ is the centroid location of the LAZ derived using the parallel axis theorem $[31,34,35]$, and together with equation 2 gives the average strain in this region. The locations of each region centroid along the $y$-direction are calculated from the neutral line height, which is in turn derived from the cross-sectional size and elastic modulus of each layer. Using the information given in Fig. 3, the neutral line height may be calculated with the following formula [31,34,35]:

$$
\mathrm{h}=\frac{\mathrm{E}_{1}\left(\mathrm{t}_{\mathrm{b} 1}^{2}+2 \mathrm{t}_{\mathrm{b} 1} \mathrm{t}_{\mathrm{laz}}+2 \mathrm{t}_{\mathrm{b} 1} \mathrm{t}_{\mathrm{b} 2}+\mathrm{t}_{\mathrm{b} 2}^{2}\right)+\mathrm{E}_{2}\left(\mathrm{t}_{\mathrm{laz}}^{2}+2 \mathrm{t}_{\mathrm{b} 2} \mathrm{t}_{\mathrm{laz}}\right)}{2\left(\mathrm{t}_{\mathrm{b} 1} \mathrm{E}_{1}+\mathrm{t}_{\mathrm{b} 2} \mathrm{E}_{1}+\mathrm{t}_{\mathrm{laz}} \mathrm{E}_{2}\right)}
$$

where $t_{b l}, t_{b 2}$ and $t_{l a z}$ are the centroid heights of the unmodified bulk regions and laser-modified region respectively. $E_{1}$ is the bulk Young's modulus for fused silica $(71.7 \mathrm{GPa})$ and for $E_{2}$ an average value of $74.75 \mathrm{GPa}$ was taken from [37] for laserdensified silica. The location of each region centroid can then be calculated from the neutral line height, as follows:

$$
\mathrm{y}_{\mathrm{b} 1}=\mathrm{t}_{\mathrm{b} 2}+\mathrm{t}_{\mathrm{laz}}+\frac{\mathrm{t}_{\mathrm{b} 1}}{2}-\mathrm{h}, \mathrm{y}_{\mathrm{b} 2}=\frac{\mathrm{t}_{\mathrm{b} 2}}{2}-\mathrm{h}, \text { and } \mathrm{y}_{\mathrm{laz}}=\mathrm{t}_{\mathrm{b} 2}+\frac{\mathrm{t}_{\mathrm{laz}}}{2}-\mathrm{h}
$$

\section{RESULTS}

\section{A. Morphology of the LAZ}

Observations of the morphology of the LAZ show a distinct trend that closely follows exposure conditions, evolving from smooth filaments for low-energy depositions to complete breakup of the laser pulse at higher exposures. The full range of pulse energy used in this experiment lies solidly above the critical power for self-focusing, as defined by $[2,4,5]$ :

$$
\mathrm{P}_{\mathrm{cr}} \equiv \frac{3.77 \lambda_{0}^{2}}{8 \pi \mathrm{n}_{0} \mathrm{n}_{2}}
$$

where $\lambda_{o}$ is the laser wavelength in vacuum, $n_{o}$ is the refractive index of silica at $\lambda_{o}=800 \mathrm{~nm}$, and $n_{2}$ is the nonlinear refractive index taken from [38]. Plugging in the numbers, equation 5 predicts the critical power to be $2.75 \pm 0.51$ MW. Comparatively, the pulse energies used in this experiment lie in the range of $\sim 9.26$ to $111 \mathrm{MW}$. 
This range of peak powers correlates closely with the observed morphology, and more interestingly, covers a wide range of features commonly associated with nonlinear pulse propagation, such as filamentation [2,4,39-41] temporal and spatial pulse splitting [42-44], multiple re-focusing cycles [45-52] and complete pulse breakup [53-61]. SEM images of these features are shown in Fig. 3, where we have highlighted some of the more prominent types of modifications along with the pulse energy, translation speed, and number of pulses per focal waist. These progressive 'snapshots' show the evolution of nonlinear propagation effects resulting from single to multi-pulse interaction with the fused silica substrate.

The simplest of these modifications, shown in Fig. 3(a-b), is a single smooth filament devoid of any additional internal structure. This type of feature is typically found for low pulse energies between 0.25 and $0.75 \mu \mathrm{J}$, with a typical width of a few hundred nanometers. For $0.25 \mu \mathrm{J}$, some evidence of the balance between self-focusing and defocusing is evident from periodic variation in width along the modification. Filaments such as these are found for higher pulse energies, but with the exception that additional structure is found at the 'head' of each modification, appearing as a series of alternating voids and spheroids. We also find 'shadow' copies of the original feature, appearing in the background as a faint but misaligned secondary pulse. It is unclear if this feature is a result of the primary structure, or simply material that has been modified by the preceding pulse. Though the listed pulse overlap is $\sim 5$ pulses / waist, the feature diameter in this case is only several hundred nanometers. Considering that the measured beam waist was $3 \mu \mathrm{m}$, technically speaking, these features still lie in the single-pulse exposure regime.

As the pulse overlap is increased, nonlinear propagation effects become more readily apparent, as evidenced by the observation of multiple foci within the LAZ, indicating stronger multi-pulse interaction and self-focusing. Damage tracks for pulse energies of $0.75 \mu \mathrm{J}$ and above are characterized by large volumes of damage, appearing as etched voids in Fig. 3(e-f) and coincide with the location of each focus. Narrow grating structures with a period of $\lambda_{o} / 2 n$ are found between each void $[43,45,62,63]$, suggesting a reflection and self-interference with the forward propagating pulse. It is unclear if this is a direct result of axial temporal splitting or just simply reflection from the generated plasma. These effects may also be explained as the result of modulational instability experienced by the propagating filament $[42,43]$. Some grating structures are found offaxis, also hinting at the possibility of additional pulse breakup and filamentation in the reverse direction [46-49,51,52,64].

The focal regions themselves are subject to high stress, resulting in localized cracking, as shown by the cleanly etched perpendicular lines projecting from each focal region (see Fig. 3(f)). Within this etched void, we observe a small spheroid of un-etched material, further suggesting the presence of intense pressure within the silica matrix. The transition from previous structures (smooth filaments with hotspots) to multiple foci is unclear, however we theorize that these structures may follow the same mechanisms as reported for the erasure of nanogratings in silica glass [65]. One possible explanation is that the hot- 
spots generated for lower pulse overlap but higher pulse energy act as seed regions, providing additional perturbation and field enhancement for the next incoming pulse to initiate further beam collapse $[45,66]$.

At the maximum exposure rate of 75 pulses per waist and pulse energies of $1.5 \mu \mathrm{J}$ and above, the latter two foci found in Fig. 3(f) merge as shown in Fig. 3(g-h), while the 'head' of the damage track becomes greatly enlarged. This merge may be due to the fact that for high laser doses, the plasma in the second hot spot cannot sufficiently attenuate the pulse. Subsequently, the area behind the hot spot is not completely protected by plasma de-focusing and is also modified. Here we observe heavy damage and disorganization, with only small evidence of counter-propagating pulses and filamentation. The 'tail' of the track remains largely unmodified, as the energy of the pulse has been significantly depleted at this stage of propagation [2,45]. Similar results have been obtained by other authors, both through simulation of filament propagation and imaging of damage in bulk fused silica $[39,41,62,63,67]$.

It is interesting to note that the multiple foci observed here are more prominent with increased pulse overlap. This may be explained by the fact that a nonlinear propagating beam has the capacity to reconstruct after an obstacle (self-healing) $[2,68,69]$. In this case, with the exposure of a small volume to multiple pulses, the incoming beam can interact with locally transformed material, which after the initial pulse has different material properties than the surrounding bulk substrate. This interaction can then lead to an increase of foci and / or damaged local zones due to the accumulation of increasing energy doses and the capacity (total energy) of the self-filamenting beam to propagate in a heavily perturbed material zone.

The majority of the structures shown in Fig. 5 were written with the beam polarization parallel to the writing direction. This orientation, which we will discuss in the next section, was also used to modify the cantilevers for volume change measurements, as these types of laser modifications have been shown to be anisotropic with polarization for both volume expansion and densification regimes $[11,12,36]$. Since this anisotropy is ever present in laser writing in one form or another $[12,70]$, we additionally explored the microstructure of modifications written with perpendicular polarization.

Features written with perpendicular polarization generally follow the same trends as for parallel, however differences in the underlying structure are evident for nearly all pulse energies and exposure conditions, as shown in Fig. 3(i-k). Typical 'hot spot' filaments are found for low pulse-overlap exposures (not shown), however increased deposition resulted in the formation of cone-like pulse breakup (Fig. 3(i)), as well as the onset of nanograting formation (Fig. 3(j-k)).

The reasons for these differences despite identical exposures are still unknown, and one could argue that each shot of the laser will have an anisotropic feature depending on the orientation of the laser field, i.e. the polarization. This anisotropy will influence the propagation and subsequent pulses in the glass, giving rise to the significant difference in structures written with 
two different polarization states. In the case of the features written here however, the polarization was carefully controlled (either perpendicular or parallel to the writing direction). Other factors such as pulse-front tilt or interplay between focal distortions (i.e. astigmatism or other off-axis effects) may have also played a major role in the difference between structures that should otherwise be identical in an isotropic material. Several authors have shown that fluctuations in laser intensity and noise $[53,54,56]$ are possible initiators of self-focusing effects. Additionally, any ellipticity present in the focus may also be responsible [59].

FIG. 3. Examples of the different morphologies found within the LAZ, classified into four groups that we have labeled Regions $1-4$. Region 1 (a-b) consists of a smooth, continuous filament. Region $2(c-d)$ resembles Region 1, but with extra structure found in the 'head' of each modification. In regions 3 and 4 , we find evidence of multiple foci $(\mathrm{e}-\mathrm{f})$ and ultimately pulse breakup with heavy material damage

$(g-h)$. Note that the images shown in $(a-f)$ are for polarization parallel to the writing direction. Some of the features found for perpendicular polarization $(\mathrm{i}-\mathrm{k})$, but with similar parameters to regions 1 and 2 , break from this convention. The writing direction for each feature shown in (a-k) is into the page, with the polarization direction given by the vector denoted $E$, and the beam propagation direction $k$.

We have classified these morphological changes into four distinct regions, as shown in Fig. 4. Each region corresponds to a distinct morphological type, with the complexity increasing with region number. Here the observation window ranges from less than 5 to nearly 80 pulses per focal waist, and in conjunction with Fig. 3 allows direct visualization of the evolution of LAZ microstructure as a function of increasing pulse number. Note that the marker size is a log scale of the deposited energy for each exposure condition.

In general, the classification is as follows: region 1 (green cross-hatch) encompasses the exposure regime where simple, smooth filaments are found (no additional structure), and is only present for the pulse energy of $0.25 \mu \mathrm{J}$ and the full range of pulse overlap. Region 2 (blue, diagonal upward-left fill) extends region 1, but for filaments with hotspot formations and small voids. This region is only found for exposure rates of 5 pulses per focal waist or less. Region 3 (yellow, vertical/diagonal fill) delineates the boundary between single- and multi-pulse interactions, and is characterized by formations containing multiple foci. This region, like region 2 , is only found for pulse energies above $0.25 \mu \mathrm{J}$, but for depositions of $\sim 19$ pulses / waist only.

FIG. 4. General classification of the morphology found within the LAZ as a function of pulse energy and the number of pulses per focal waist. We define each region according to the structure observed, with region 1 and 2 corresponding to a single smooth filament, with the exception that for region 2, filaments are found to have additional structure within the 'head' of the feature. Regions 3 and 4 correspond to 
the onset of multiple foci, with region 4 corresponding to strong pulse breakup and heavy material damage. Note that marker size is shown on a logarithmic scale (see legend at right) and corresponds to the deposited energy for each exposure condition.

Finally, region 4 (red, diagonal upward-right fill) describes all features found at the highest exposure rates of 75 pulses per waist, and is characterized by heavy damage and disorganization within the focal volume. While this classification does not provide a complete picture, it nevertheless serves as a useful guide. In these experiments, this set of morphological classifications generally only applies to morphologies observed for the writing polarization oriented parallel to the writing direction.

FIG. 5. Examples of the repeatability of writing with pulses in the sub- $30 f s$ regime. $E$ denotes the beam polarization direction, $s$ the travel direction of the focus during writing, and $k$ the propagation direction of the beam.

One could argue that the results presented in Fig. 5 represent the best-case scenario of features generated using laser pulses below $30 \mathrm{fs}$, however this is not the case. Despite operating well within the unstable regime of nonlinear propagation effects, the features obtained from the lines sample were highly repeatable, as shown in Fig. 3 for both low and high pulse energies. The results for $0.25 \mu \mathrm{J}$ are perhaps not so surprising, since in this case we are relatively close to the critical threshold for selffocusing, so the effects of nonlinear propagation are less evident other than the formation of continuous filaments. Further increase of the pulse energy again results in stronger interaction, however the structures appear to form with nearly the same regularity for every exposure instance. Note that these lines (shown in Fig. 3 and 5 as an end-facet view, translation direction into the page), were written sequentially from left to right, and verified against limited cross-sections of the machined cantilevers, which contained many closely spaced lines. Though we have only highlighted a few cases here, all exposures exhibited the same repeatability, demonstrating the potential applicability of this sub-30 $f s$ writing process to laser-based fabrication techniques in bulk transparent materials.

\section{B. TEM observations}

Thin sections were investigated using a Transmission Electron Microscope (TEM, Tecnai Osiris) operating at $200 \mathrm{kV}$. Scanning TEM (STEM) mode was used for imaging, taking advantage of both bright field (BF) and high-angle annular dark field (HAADF) detectors (see Fig. 6 and 7). 
Figure 6 (3): STEM images of thin section TS1 in bright field (a) and HAADF (b) imaging modes. Glass modifications in this section correspond to an exposure of $3 \mu \mathrm{J} /$ pulse with a velocity of $16 \mu \mathrm{m} / \mathrm{s}$. See Fig. 2 for a layout of the sectioned area in relation to the lasermodified regions.

Figure 7: STEM images of thin-section TS2 in bright field (a) and HAADF (b) imaging modes. Glass modifications in this section correspond to an exposure of $3 \mu \mathrm{J} /$ pulse and a velocity of $4 \mu \mathrm{m} / \mathrm{s}$.

In TS1, we observe a porous morphology of the glass (Fig. 6), which is a result of the brief post etch after writing (as shown in Fig. 3, 5 and 8 and outlined in Section II, part B.). Aside from this large-scale porosity, no other unique morphology was found, for example, such as that observed in [71,72]. Additionally in thin section TS2, taken as a cross-section from the tails of the laser-modified regions, we find the same lack of additional structure, even when using high-magnification TEM imaging as shown in Fig. 7. This may indicate a region that has been significantly densified and of a different nature than material usually found in nanogratings [71,72].

Further investigations were conducted using electron diffraction to search for possible crystalline phases. Here the expectation was to observe crystalline phases due to high the energy load and pressures generated during femtosecond irradiation, and formation of new phases upon cooling. For instance, Gamaly et al. [73] mentioned that quartz and fused silica could convert to a dense phase of stishovite in the range of $15-46 \mathrm{GPa}$; transient pressures easily achieved with femtosecond irradiation. Unfortunately, both samples showed no sign of crystalline $\mathrm{Si}$ or $\mathrm{SiO}_{2}$, as the modified volumes are extremely small, making detection using electron diffraction difficult. It remains difficult however to conclude that no metastable crystalline phases were present prior to sample processing, as sample preparation of thin sections remains a highly invasive technique.

\section{Calculated strain}

Following the derivations laid out in Section IIC, we now focus on the calculation of cantilever strain from the measured deflection and compare it with the observed microstructure found within the LAZ. This data is summarized in Fig. 8, and plotted against deposited energy in order to make a comparison with previous work [36]. The data in Fig. 8(a) shows an overall trend of increasing strain for increasing deposited energy, and is most evident for the two lowest pulse energies of 0.25 and $0.75 \mu \mathrm{J}$, the latter of which achieves the highest level of average strain at $0.008 \%$. This trend however, falls short for pulse energies above $0.75 \mu \mathrm{J}$, becoming chaotic after reaching a threshold of $\sim 0.5 \mathrm{~J} / \mathrm{mm}^{2}$. When comparing similar values of deposited 
energy (for example, $\sim 2 \mathrm{~J} / \mathrm{mm}^{2}$ at 0.75 and $3 \mu \mathrm{J}$ ), we find that seemingly equivalent values do not correlate with the expected outcome, suggesting that the measure of incident deposited energy does not sufficiently capture all material modification effects in this repetition rate regime.

FIG. 8. (a) Plot of average strain versus deposited energy, with trend lines shown for clarification. The highlighted points $(1-4)$ are shown in (b), where SEM imaging reveals the underlying microstructure for $(1,2) 0.75 \mu \mathrm{J}, 4$ and $16 \mu \mathrm{m} / \mathrm{s}$, and (3,4) $2.25 \mu \mathrm{J}, 4$ and $16 \mu \mathrm{m} / \mathrm{s}$, respectively. For equivalent translation speeds, these features are exposed to the same number of pulses per focal spot (75 and 18.75 shots for 4 and $16 \mu \mathrm{m} / \mathrm{s}$ respectively), but exhibit vastly different morphology. For $0.75 \mu \mathrm{J}$ the laser-induced damage is relatively smooth and free of complex structure, while those for $2.25 \mu \mathrm{J}$ are highly structured, exhibiting signs of pulse filamentation and regions of heavy modification. In (4), the top-most (closest to the incoming laser pulse) region shows signs of crack propagation perpendicular to the modification direction. This cracking is likely the cause for the chaotic behavior seen in (a) for pulse energies above $0.75 \mu \mathrm{J}$. Note that these images were acquired from the secondary lines sample then later confirmed against cross-sectional microscope images of the cantilevers.

These differences are most apparent when viewing the microstructure formed within the LAZ, as shown in the SEM images in Fig. 8(b) for two different exposure regimes. Here, we compare moderate extremes: $0.75 \mu \mathrm{J}$ (the threshold for 'chaotic deflection') and $2.25 \mu \mathrm{J}$. These pulse energies best illustrate the differences in structure for an equivalent number of pulses per focal waist. Beginning with the pulse energy of $0.75 \mu \mathrm{J}$, shown for the speeds of 4 and $16 \mu \mathrm{m} / \mathrm{s}$ as indicated by the callouts 1 and 2 respectively, we again observe a relatively smooth and homogeneous filament, with only slight irregularities. This structure is in sharp contrast to that found for the pulse energy of $2.25 \mu \mathrm{J}$ (callouts 3 and 4 respectively, same speeds), which, as we have discussed in the previous section, is the result of nonlinear propagation effects. In this case, the multiple foci are also regions that were subject to high energy density. This is evident by the presence of small un-etched spheroids, suggesting highly densified material. A side effect of this energy concentration can also be seen at the peripheral of the initial focus, where two cracks are found perpendicular to the pulse propagation axis. From a micro-mechanical point of view, it is easy to see which structure is more conducive to producing a large deflection at high values of laser exposure.

While the disparity in structure for these two pulse energies explains the differences in the calculated strain, it does not necessarily mean that higher strain was not obtained, but simply that crack formation is a limiting factor when using this measurement technique. Nevertheless, we conclude that more energy per pulse is not necessarily better when machining. In this case, an accumulation of low-energy pulses is more beneficial to the machining process. The resulting structures are 
smooth, highly densified, and due to the lack of large 'defects' within the structure itself, resistant to localized crack formation and failure. At higher energies and deposition rates, nonlinear pulse interaction with the substrate leads to the generation of complex structures, increasing the likelihood of stress relief through crack formation. Similar structures were found for the pulse energies of 1.5 and $3 \mu \mathrm{J}$.

\section{D. $\mu$-Raman maps of the LAZ}

Structural changes at the molecular level may also be correlated to the observed strain through the use of Raman microscopy. In particular, we focus on the $D_{1}$ and $D_{2}$ peaks, which correspond to the statistical distribution of $\mathrm{N}=3$ and $\mathrm{N}=4$ membered rings within the silica matrix [16,25,26,74]. A reduction in ring order, brought about through laser exposure, generates a change in peak intensity with respect to the bulk. This reduction is thought to be a key indicator for densification in silica glass $[18-21,23,24]$. Here, we chose to generate Raman maps of each LAZ, as the peak height and shift may be spatially located within the LAZ.

The blue and red curves shown in Fig. 9(a) illustrate typical Raman spectra for bulk and laser-modified silica respectively, shown for an exposure of $750 \mathrm{~nJ}, 4 \mu \mathrm{m} / \mathrm{s}$ with prominent peaks $\left(\omega_{1}, D_{1}, D_{2}\right.$, and $\left.\omega_{3}\right)$ labeled for clarity. The unmodified spectrum was taken from a point far outside the LAZ (lower left of the inset image) while the shifted spectrum is from the region within the LAZ that has been heavily modified. Upon first examination, the shifted spectrum shows all the characteristic signs of material densification: the main band $\left(\omega_{l}\right)$ has narrowed and shifted to a higher wavenumber, with an increase of the peak intensity. Accordingly, an increase of the $D_{1}$ and $D_{2}$ peaks is also found. Indeed, an increase of the $D_{2}$ peak is indicative of the occurrence of 3-membered rings, suggesting a reduction in ring order within the glass matrix, leading to overall compaction of the material within the LAZ [17-19,21,23,24,72].

FIG. 9 (a) Example Raman spectra from modified and un-modified regions of the silica substrate for comparison. (b) Plot of average strain along with intensity of the $D_{2}$ peak as a function of deposited energy for a pulse energy of $0.75 \mu \mathrm{J}$. The vertical error bars represent the measurement uncertainty and standard deviation for the calculated strain and $D_{2}$ peak intensity respectively, while the horizontal error bars (strain) represent uncertainty of the deposited energy based on the variation of the width of the LAZ. Here the Raman curves have been normalized using the $\omega_{3}$ band. Note that in (a), the spectrum shown for the LAZ region is partially obscured by the curve for the bulk to the left of the main peak. Despite the appearance of being wider, this peak is in fact slightly narrower than the main band for the un-modified region when measured at $70 \%$ of the peak height for each curve, respectively. 
The curves shown in Fig. 9(b) represent the measured intensity of the $D_{2}$ peak (green circles, left axis) and the calculated strain (blue squares, right axis). Similar to the spectra shown in Fig. 9(a), the $D_{2}$ peak intensity was extracted from the center of the Raman map for each LAZ, which are shown in the false-color images below the curve. For comparison, a cross-sectional microscope image of each LAZ is also shown, giving the spatial location of the strongest intensity change in the Raman peaks. Note that these two curves are shown on the same graph to illustrate a (qualitative) correlation, i.e. the intensity of the $D_{2}$ peak cannot be quantitatively related to the level of densification achieved for a given exposure condition, but nevertheless shows the same trend as the strain curve. From this set of curves, we can see a clear trend relating the increase of material strain to the change of the $D_{2}$ peak intensity.

Caution should be taken however when interpreting this information, particularly with regards to the morphology of the LAZ and the spatial extent of the $D_{l}$ and $D_{2}$ maps. Aside from this writing condition $(750 \mathrm{~nJ}, 4 \mu \mathrm{m} / \mathrm{s})$, no observable changes were found for the $D_{l}$ peak at lower deposited energies. Furthermore, the change in $D_{l}$ is only located within the heavily modified region of the LAZ shown in the inset of Fig. 9(a). These observations are especially prominent when making a comparison between $D_{1}$ and $D_{2}$, as the map for $D_{2}$ extends well into the smooth region above the focus. A similar trend to that of $D_{l}$ is also found for the $\omega_{l}$ peak, which is again only located within the heavily modified region, but not present for lower deposited energy. These differences of spatial location and lack of changes at lower deposited energy suggest the presence of broken bonds within the heavily modified region. This region is likely still densified (as indicated by the increase in $D_{2}$ ), however the broken bonds appear to contribute to an increase in the etching rate, as evidenced by the rough morphology shown in the SEM image of Fig 5(b), callout 2.

\section{E. Effect of writing direction}

Though the analytical model covered in Section IIC is somewhat simplistic, it nevertheless serves as a useful metric to compare average strain between this set of experiments and those previously published [36]. Average strain, and not the absolute strain, was chosen due to the fundamental differences between the two experiments. We justify this choice by examining the stress, and ultimately the strain, between the laser-written lines using stress-induced birefringence, as shown in Fig. 10.

FIG. 10. (a) Plot of the average strain derived from retardance measurements for various writing conditions. The inset shows a typical line profile (red) and the associated curve fit (orange) taken across the retardance peaks of each measurement, which are shown in green. The inset example is taken from the four peaks shown in (b). (b-e) retardance maps corresponding to the same exposures as shown for the SEM images in Fig. 3. Note that the retardance maps are quite complex, even for the 'best case' exposure conditions that yield maximum strain

$$
\text { (i.e. } 0.75 \mu \mathrm{J}, 4 \mu \mathrm{m} / \mathrm{s} \text { ). }
$$


Similar to the plot shown in Fig. 8(a), the strain increases with increasing deposited energy, however a noticeable break is found for deposited energies above $1 \mathrm{~J} / \mathrm{mm}^{2}$. This threshold again corresponds to the onset of highly disorganized structure within the LAZ. However, as indicated in the images shown in Fig. 10(b-e), the distribution of strain between the lines is not homogeneous, suggesting that while the localized strain may be higher, the overall assumption of average strain remains reasonable.

It is important to note here that we are only interested in the birefringence generated between the lines, as any measurements from the lines themselves contain retardance information of both the internal strain as well as the total refractive index change within the LAZ. As these two pieces of information are inseparable, this data does not provide meaningful information about the overall strain in the substrate.

Figure 11. A comparison of line orientation during the writing process for the same exposure conditions. The upper curve represents the writing geometry used in [36], with lines written perpendicular to the long axis of the cantilever. The lower two curves are for the method used in this work (lines parallel to the long axis), with two different physical orientations of the beam with respect to the focus (but with the same line orientation within the cantilever). This was done to check for anisotropy in the laser focus. The difference in predicted strain ranges from $50 \%$ for high values of $\mathrm{E}_{\mathrm{d}}$ up to $1000 \%$ for low values, suggesting that parallel writing provides greater measurement sensitivity for low values of induced strain.

One takeaway that is important to remember is that we are creating a composite structure. As such, physical parameters such as line density, location with respect to the surface, and orientation of the written lines will all play a role in the outcome of the measured strain. Though the model we have used compensates somewhat for the depth of the written structures within the substrate, it does not take into account the orientation of the lines. The impact of different line orientation, and hence the measured strain, is displayed in Fig. 11.

Typically, as in [36] we have employed the writing scheme shown in the upper left of Fig. 11, where modifications are written perpendicular to the long axis of each cantilever. This 'accordion'-like structure provides several benefits: the impact of varying line spacing, depth, and exposure are readily apparent in the measurement outcome. Additionally, through several basic assumptions about the nature of the material change within the LAZ, we can make inferences about the absolute strain within each modified line. Here however, due to limitations in the writing setup, lines were written parallel to each cantilever 
(parallel to the long axis), similar to the schemes shown below the curves in Fig. 11. This comparison was performed using the same exposure with a pulse energy of $160 \mathrm{~nJ}$ at $\sim 300 \mathrm{fs}$ (volume expansion regime) and identical cantilever structures as those used in this work, and shows a clear difference between the two measured strains. With our current writing scheme, the strain is under-predicted by as little as $50 \%$ for values of deposited energy above $2 \mathrm{~J} / \mathrm{mm}^{2}$, and as much as $1000 \%$ at $1 \mathrm{~J} / \mathrm{mm}^{2}$, meaning that perpendicular writing is especially sensitive to small changes in density as compared to parallel writing.

\section{F. Refractive index calculations}

From this data we can calculate $\Delta n$ for comparison to the results presented in [36], however this value will be limited to the 'composite' case: we must consider that we are computing an average despite the fact that the region of interest is a composite material. In this example, the single-line $\Delta n$ (and strain) is likely higher; however the model used here is limited as other factors are at work, such as inhomogeneity and orientation of the written lines with respect to the cantilever geometry.

We compute an estimated $\Delta n$ following the procedure outlined in [36], taking a modified form of the Lorenz-Lorenz relation $[24,75,76]$ :

$$
\Delta \mathrm{n}_{\lambda}=\left[\frac{\left(\mathrm{n}_{\lambda}^{2}-1\right)\left(\mathrm{n}_{\lambda}^{2}+2\right)}{6 \mathrm{n}_{\lambda}}\right]\left(\frac{3 \varepsilon_{\mathrm{laz}}}{1+2 v}\right)(1+\Omega)
$$

where $n_{\lambda}$ is the refractive index of silica glass at $633 \mathrm{~nm}$, Elaz is the average strain for a given exposure condition, and $v$ is the Poisson coefficient for fused silica. The value of $\Omega$ was taken from [24] for UV densified silica, which was measured to be in the range of $-0.23 \pm-0.04$. For simplicity, we assume that the strain within the LAZ is isotropic and take the maximum value of $7.8 \times 10^{-5}$ obtained for $0.75 \mu \mathrm{J}$. These values, taken without error compensation for writing orientation, result in an estimated change of refractive index of $\Delta n_{\|}=7.21 \times 10^{-5} \pm 7.5 \times 10^{-6}$. If we assume that the strain is under-estimated by $50 \%$ as shown in Fig. 11, this value increases to $\Delta n_{\|}=1.02 \times 10^{-4} \pm 1.12 \times 10^{-5}$, which begins to approach the range of significantly useful refractive index change. This equates to an average densification of approximately $0.017 \%$, or roughly the same level as reported in [36]. Note that the values reported in [36] are for a compensated 'absolute' strain, taking into account the localized strain between the LAZ and unmodified material. We must stress here that this measurement is an average, and that locally the value for $n$ is likely much higher.

\section{DISCUSSION}

Recently, we have investigated densification effects in fused silica for the regime corresponding to 150 fs pulses, specifically to study the effects on the refractive index and stress state when the material is subject to varying exposure 
conditions [36]. This work shows that the stress state within the substrate can be continuously tuned from compression to expansion depending on the exposure conditions used. In the densification regime, we observed a strong correlation between maximum densification and sufficient index change to support waveguiding. However, the maximum attainable densification, and hence the largest $\Delta n$, is limited: simply increasing the exposure rate leads to the generation of nanostructures within the LAZ, resulting in poor mode confinement and higher losses. Ultimately, these nanostructures develop into nanogratings and the stress state inverts, though it should be noted that this inversion can also be evidence of porosity in the LAZ [36,71]. No guiding is found in this regime. This work hints at the possibility of improved densification at shorter pulses, leaving pulsewidth as the final tuning parameter.

In our present work, we find the same general outcome as that presented in [36]: an increase of the exposure rate does not necessarily yield higher densification, often leading to stress relief through cracking. It should be noted however that the mechanisms involved are fundamentally different.

To summarize, our measurements show that:

- The rate of energy deposition is highly influential on the outcome of the material state after laser exposure: identical cumulative energy dosages (more pulses at low energy versus fewer pulses at high energy) do not necessarily yield the same outcome. In general, for our chosen exposure range, lower pulse energy is found to be beneficial to densification, however an increase of the deposition rate leads to microstructure generation within the LAZ and cracking for all but the two lowest pulse energies used. This ultimately serves as the limiting factor for measuring higher densification in this experiment. This lack of 'energetic equivalence' suggests that the definition of deposited energy should be modified when working in this exposure regime.

- Short and low pulse exposure can lead to well-defined densified zones, despite the highly nonlinear regime of interaction, and can be repeatedly achieved with the same exposure parameters. The level of densification is at least as good as that obtained with $150 \mathrm{fs}$ pulse duration, and possibly higher, achieving an average densification of $0.017 \%$, or approximately the same order of magnitude as that reported in [36]. Note that the values given in [36] are for a compensated strain, taking into account the absolute strain of the LAZ itself, which is typically higher than the average as reported here. If we take this into consideration, and include the corrections given in Fig. 11, then the values obtained for this experiment are significantly better.

- The maximum measurable strain is limited, at least in this case, using the cantilever deflection technique. This limit is a function of both the writing conditions used and the manner in which the laser-written structures are created. 
- Despite these limitations, the results show strong evidence that highly densified material (beyond what was measured) may be present. Examining the data for the highest strain at $0.75 \mu \mathrm{J}$, we find that despite a continuously increasing strain for this exposure (i.e. no evidence of unpredictable deflection), structural 'defects' are still found within the LAZ (see images, Fig. 8 and 9). Furthermore, the Raman maps shown in Fig. 9 show a distinct lack of localized intensity reduction that would indicate severe lattice damage, suggesting that not all defects are necessarily related to stress relief. Here however we must be careful in the definition of what features constitute defects in terms of the desired outcome of this experiment.

- The observed cracking in the chaotic deflection regime for higher values of deposited energy appears to be a secondary effect of highly localized stress (see Fig. 10), further supporting the hypothesis that the strain at this exposure level is indeed higher than the measured average value.

- In this particular set of exposure conditions, we observed a broad range of structural modifications as a result of nonlinear propagation effects such as filamentation, hot spot formation, multiple foci, and axial as well as lateral pulse splitting, both spatial and temporal. These structures manifest in a variety of combinations, resulting in highly complex structures, which despite these complexities are nevertheless repeatable and as such, potentially useful for applications in laser materials processing.

\section{CONCLUSION}

We have reported on the variety of microstructures that are found as a result of exposing fused silica to low-energy, i.e. peak irradiance levels of $<3.710^{12} \mathrm{~W} / \mathrm{cm}^{2}$, sub-30 fs focused laser pulses. Although the nature of the nonlinear interactions found in this regime has been a discouraging factor, we show here that stable and well-defined laser-modified zones can be achieved, producing a significant level of densification. In particular, this approach offers a potential tool for inducing higher strain in fused silica. Using a method based on cantilever deflection, we find a level of strain that is roughly the same order of magnitude as compared to previous experiments [36]. Here the limit in measuring higher strain is linked to the measurement method rather than flaws within the LAZ itself, and our data shows that higher strain than what was measured may still be present. Data obtained from SEM images shows that the measurement limits are related to the structure generated within the LAZ during processing.

Overall, better results were obtained with an accumulation of low-energy pulses over a longer period of time, and the use of higher energy pulses is more likely to induce structural modifications that enhance self-focusing effects. In this case, the structures generated often show signs of highly localized strain, leading to secondary cracking of the surrounding material. 
This data, combined with micro-Raman maps of select LAZ regions, give strong evidence that not all observed structure is related to stress relief. We also find that writing direction with respect to the cantilever long axis plays an important role in the measurement of strain using the deflection method. In our case, the chosen writing direction results in an under-estimation of the strain between 50 and $1000 \%$, for high versus low deposited energy respectively, suggesting that writing direction also determines the sensitivity of the measurement. Generally, we find that two distinct factors play a role in the processing outcome: nonlinear propagation effects, as well as the interaction between an incoming pulse and the byproduct of its predecessors (spatially cumulative effect)

Different results are obtained through different illumination strategies, and this approach can be used to mitigate nonlinear effects. Using this low-energy pulse accumulation strategy, homogeneous modifications were obtained, despite being above the critical power for self-focusing. These homogeneous modifications are directly responsible for the highest measured densification presented in this work. Beyond this homogeneous regime, we observed a wealth of interesting and repeatable structural modifications, which highlight the various phenomena found in nonlinear propagation including filamentation, formation of multiple foci, and temporal and spatial pulse splitting. We show that these structures, while highly complex, are repeatable, highlighting their possible use in various applications in laser processing of bulk dielectric materials such as waveguide fabrication. We hope that these novel experimental results, which are the direct outcome of these complex interactions, will help refine models and simulations that have been performed to investigate the propagation of ultrashort laser pulses.

\section{Acknowledgements}

This project was partially funded thorough support from LaserLab Europe (Project Laserlab-Europe EU-H2020 654148 \& 871124) and was done in the context of the European Research Council project 'Galatea' (ERC-Stg, ERC-2012-StG307442). Part of this work was also supported by the Swiss National Science Foundation (FNS 200021 169681). The Galatea Lab further acknowledges the sponsoring of Richemont International.

\section{Data availability}

The data that support the findings of this study are available from the corresponding author upon reasonable request.

1. A. Dubietis and A. Couairon, Ultrafast Supercontinuum Generation in Transparent Solid-State Media (Springer, Cham, 2019).

2. A. Couairon and A. Mysyrowicz, "Femtosecond filamentation in transparent media," Phys. Rep. 441, 47-189 (2007).

3. K. M. Davis, K. Miura, N. Sugimoto, and K. Hirao, "Writing waveguides in glass with a femtosecond laser," Opt. Lett. 21, 1729 (1996).

4. J. H. Marburger, "Self-focusing: Theory," Prog. Quant. Electron. 4, Part 1, 35-110 (1975). 

by tightly focused femtosecond laser pulses," Meas. Sci. Technol. 12, 1784-1794 (2001).

6. R. Graf, A. Fernandez, M. Dubov, H. J. Brueckner, B. N. Chichkov, and A. Apolonski, "Pearl-chain waveguides written at megahertz repetition rate," Appl. Phys. B-Lasers O. 87, 21-27 (2007).

7. C. Hnatovsky, R. S. Taylor, P. P. Rajeev, E. Simova, V. R. Bhardwaj, D. M. Rayner, and P. B. Corkum, "Pulse duration dependence of femtosecond-laser-fabricated nanogratings in fused silica," Appl. Phys. Lett. 87, 014104-3 (2005).

8. O. Uteza, P. Blandin, L. Charmasson, G. Coustillier, D. Grojo, A. Kabashin, M. Lebugle, N. Sanner, V. Tcheremiskine, M. Sentis, F. Légaré, and J. C. Kieffer, "ASUR : plateforme d'applications des sources laser ultrarapides pour l'imagerie X et l'interaction laser-matière," in E. Constant, P. Martin, and H. Bachau, eds. (EDP Sciences, 2013), Vol. 94, p. 01004.

9. S. Rajesh and Y. Bellouard, "Towards fast femtosecond laser micromachining of fused silica: The effect of deposited energy," Opt. Express 18, 21490 (2010).

10. A. Champion and Y. Bellouard, "Direct volume variation measurements in fused silica specimens exposed to femtosecond laser," Opt. Mater. Express 2, 789-798 (2012).

11. A. Champion, M. Beresna, P. Kazansky, and Y. Bellouard, "Stress distribution around femtosecond laser affected zones: effect of nanogratings orientation," Opt. Express 21, 24942-24951 (2013).

12. B. McMillen and Y. Bellouard, "On the anisotropy of stress-distribution induced in glasses and crystals by nonablative femtosecond laser exposure," Opt. Express 23, 86-100 (2015).

13. A. Marcinkevicius, S. Juodkazis, M. Watanabe, M. Miwa, S. Matsuo, H. Misawa, and J. Nishii, "Femtosecond laserassisted three-dimensional microfabrication in silica," Opt. Lett. 26, 277-279 (2001).

14. Y. Bellouard, A. Said, M. Dugan, and P. Bado, "Fabrication of high-aspect ratio, micro-fluidic channels and tunnels using femtosecond laser pulses and chemical etching," Opt. Express 12, 2120 (2004).

15. L. Sudrie, M. Franco, and B. Prade, " Study of damage in fused silica induced by ultra-short IR laser pulses," Opt. Commun. 191, 333-339 (2001).

16. A. E. Geissberger and F. L. Galeener, "Raman studies of vitreous $\mathrm{SiO}_{2}$ versus fictive temperature," Phys. Rev. B 28 , 3266-3271 (1983).

17. H. Sugiura and T. Yamadaya, "Raman scattering in silica glass in the permanent densification region," J. Non-Cryst. Solids 144, 151-158 (1992).

18. C. J. Benmore, E. Soignard, S. A. Amin, M. Guthrie, S. D. Shastri, P. L. Lee, and J. L. Yarger, "Structural and topological changes in silica glass at pressure," Phys. Rev. B 81, 054105 (2010).

19. J. W. Chan, T. Huser, S. Risbud, and D. M. Krol, "Structural changes in fused silica after exposure to focused femtosecond laser pulses," Opt. Lett. 26, 1726-1728 (2001).

20. M. Okuno, B. Reynard, Y. Shimada, Y. Syono, and C. Willaime, "A Raman spectroscopic study of shock-wave densification of vitreous silica," Phys. Chem. Miner. 26, 304-311 (1999).

21. C. Z. Tan, J. Arndt, and H. S. Xie, "Optical properties of densified silica glasses," Physica B 252, 28-33 (1998).

22. N. Kitamura, Y. Toguchi, S. Funo, H. Yamashita, and M. Kinoshita, "Refractive index of densified silica glass," J. Non-Cryst. Solids 159, 241-245 (1993).

23. S. Vukelić, P. Kongsuwan, S. Ryu, and Y. L. Yao, "Ultrafast Laser Induced Structural Modification of Fused Silica_Part II: Spatially Resolved and Decomposed Raman Spectral Analysis," J. Manuf. Sci. Eng. 132, 061013 (2010).

24. R. E. Schenker and W. G. Oldham, "Ultraviolet-induced densification in fused silica," J. Appl. Phys. 82, 1065-1071 (1997).

25. K. Awazu and $\mathrm{H}$. Kawazoe, "Strained $\mathrm{Si}-\mathrm{O}-\mathrm{Si}$ bonds in amorphous $\mathrm{SiO}_{2}$ materials: A family member of active centers in radio, photo, and chemical responses," J. Appl. Phys. 94, 6243-6262 (2003).

26. F. L. Galeener, "Planar rings in vitreous silica," J. Non-Cryst. Solids 49, 53-62 (1982)

27. B. McMillen, C. Athanasiou, and Y. Bellouard, "Femtosecond laser direct-write waveplates based on stress-induced birefringence," Opt. Express 24, 27239-27252 (2016).

28. "OpenPolScope: Home," http://www.openpolscope.org.

29. S. B. Metha, M. Shribak, and R. Oldenbourg, "Polarized light imaging of birefringence and diattenuation at high resolution and high sensitivity," J. Opt. 15, 094007 (2013).

30. M. Lekstrom, M. A. McLachlan, S. Husain, D. W. McComb, and B. A. Shollock, "Using the in situ lift-out technique to prepare TEM specimens on a single-beam FIB instrument," J. Phys.: Conf. Ser. 126, 012028 (2008).

31. S. Timoshenko, "Analysis of bi-metal thermostats," J. Opt. Soc. Am. 11, 233-255 (1925).

32. M. Benabdi and A. A. Roche, "Mechanical properties of thin and thick coatings applied to various substrates. Part I. An elastic analysis of residual stresses within coating materials," J. Adhes. Sci. Technol. 11, 281-299 (1997).

33. M. Christophersen, B. Shapiro, and E. Smela, "Characterization and modeling of PPy bilayer microactuators: Part 1. Curvature," Senor. Actuat. B-Chem. 115, 596-609 (2006). 
R. G. Budynas and W. C. Young, Roark's Formulas for Stress and Strain, 7 ed. (McGraw-Hill, 2002).

J. M. Gere and S. P. Timoshenko, Mechanics of Materials (PWS Publishing Company, 1997).

Y. Bellouard, A. Champion, B. McMillen, S. Mukherjee, R. R. Thomson, C. Pépin, P. Gillet, and Y. Cheng, "Stressstate manipulation in fused silica via femtosecond laser irradiation," Optica 3, 1285-1293 (2016).

Y. Bellouard, T. Colomb, C. Depeursinge, and M. Dugan, "Nanoindentation and birefringence measurements on fused silica specimen exposed to low-energy femtosecond pulses," Opt. Express 14, 8360 (2006).

D. Milam, "Review and assessment of measured values of the nonlinear refractive-index coefficient of fused silica," Applied Optics 37, 546-550 (1998).

A. Couairon, L. Sudrie, M. Franco, B. Prade, and A. Mysyrowicz, "Filamentation and damage in fused silica induced by tightly focused femtosecond laser pulses," Phys. Rev. B 71, 125435 (2005).

L. Sudrie, A. Couairon, M. Franco, B. Lamouroux, B. Prade, S. Tzortzakis, and A. Mysyrowicz, "Femtosecond Laser-Induced Damage and Filamentary Propagation in Fused Silica," Phys. Rev. Lett. 89, 186601 (2002).

Z. Wu, H. Jiang, Q. Sun, H. Yang, and Q. Gong, "Filamentation and temporal reshaping of a femtosecond pulse in fused silica," Phys. Rev. A 68, 063820 (2003).

S. Tzortzakis, L. Sudrie, M. Franco, B. Prade, A. Mysyrowicz, A. Couairon, and L. Bergé, "Self-Guided Propagation of Ultrashort IR Laser Pulses in Fused Silica," Phys. Rev. Lett. 87, 213902 (2001).

S. Henz and J. Herrmann, "Self-channeling and pulse shortening of femtosecond pulses in multiphoton-ionized dispersive dielectric solids," Phys. Rev. A 59, 2528-2531 (1999).

Multiple-cone formation," Phys. Rev. E 66, 056608 (2002).

M. Mlejnek, E. M. Wright, and J. V. Moloney, "Dynamic spatial replenishment of femtosecond pulses propagating in air," Opt. Lett. 23, 382-384 (1998).

N. A. Zharova, A. G. Litvak, T. A. Petrova, A. M. Sergeev, and A. D. Yunakovskii, "Multiple fractionation of wave structures in a nonlinear medium," JETP Lett. 44, 13 (1986).

N. A. Zharova, A. G. Litvak, and V. A. Mironov, "On the collapse of wave packets in a medium with normal group velocity dispersion," JETP Lett. 75, 539-542 (2002).

P. Chernev and V. Petrov, "Self-focusing of light pulses in the presence of normal group-velocity dispersion," Opt. Lett. 17, 172-174 (1992).

J. E. Rothenberg, "Pulse splitting during self-focusing in normally dispersive media," Opt. Lett. 17, $583-585$ (1992).

G. Fibich, V. M. Malkin, and G. C. Papanicolaou, "Beam self-focusing in the presence of a small normal time dispersion," Phys. Rev. A 52, 4218-4228 (1995).

G. Fibich and G. C. Papanicolaou, "Self-focusing in the presence of small time dispersion and nonparaxiality," Opt. Lett. 22, 1379-1381 (1997).

G. Fibich and B. Ilan, "Self-focusing of circularly polarized beams," Phys. Rev. E 67, 036622 (2003).

V. I. Bespalov and V. I. Talanov, "Filamentary structure of light beams in nonlinear liquids," JETP Lett. 3, 307 (1966).

Z.-Q. Hao, J. Zhang, X. Lu, T.-T. Xi, Y.-T. Li, X.-H. Yuan, Z.-Y. Zheng, Z.-H. Wang, W.-J. Ling, and Z.-Y. Wei, "Spatial evolution of multiple filaments in air induced by femtosecond laser pulses," Opt. Express 14, 773-778 (2006).

5. F. Vidal and T. W. Johnston, "Electromagnetic Beam Breakup: Multiple Filaments, Single Beam Equilibria, and Radiation," Phys. Rev. Lett. 77, 1282-1285 (1996).

G. Fibich, S. Eisenmann, B. Ilan, Y. Erlich, M. Fraenkel, Z. Henis, A. L. Gaeta, and A. Zigler, "Self-focusing distance of very high power laser pulses," Opt. Express 13, 5897-5903 (2005).

M. Mlejnek, M. Kolesik, J. V. Moloney, and E. M. Wright, "Optically Turbulent Femtosecond Light Guide in Air," Phys. Rev. Lett. 83, 2938-2941 (1999).

G. Méchain, A. Couairon, M. Franco, B. Prade, and A. Mysyrowicz, "Organizing Multiple Femtosecond Filaments in Air," Phys. Rev. Lett. 93, 035003 (2004).

A. Dubietis, G. Tamosauskas, G. Fibich, and B. Ilan, "Multiple filamentation induced by input-beam ellipticity," Opt. Lett. 29, 1126 (2004).

G. Fibich and B. Ilan, "Optical light bullets in a pure Kerr medium," Opt. Lett. 29, 887-889 (2004).

H. Schroeder and S. L. Chin, "Visualization of the evolution of multiple filaments in methanol," Opt. Commun. 234, 399-406 (2004).

M. Kolesik, E. M. Wright, and J. V. Moloney, "Dynamic Nonlinear X Waves for Femtosecond Pulse Propagation in Water," Phys. Rev. Lett. 92, 73 (2004).

63. A. Couairon, E. Gaižauskas, D. Faccio, A. Dubietis, and P. Di Trapani, "Nonlinear X-wave formation by femtosecond filamentation in Kerr media," Phys. Rev. E 73, 016608 (2006).

G. Fibich, V. M. Malkin, and G. C. Papanicolaou, "Beam self-focusing in the presence of a small normal time dispersion," Phys. Rev. A 52, 4218-4228 (1995). 
65. A. Rudenko, J.-P. Colombier, and T. E. Itina, "Nanopore-mediated ultrashort laser-induced formation and erasure of volume nanogratings in glass," Phys. Chem. Chem. Phys. 20, 5887-5899 (2018).

66. V. R. Bhardwaj, E. Simova, P. P. Rajeev, C. Hnatovsky, R. S. Taylor, D. M. Rayner, and P. B. Corkum, "Optically Produced Arrays of Planar Nanostructures inside Fused Silica," Phys. Rev. Lett. 96, 057404 (2006).

67. L. Sudrie, "Propagation non-lineaire des impulsions laser femtosecondes dans la silice," Université Paris XI Orsay (2002).

68. M. Kolesik and J. V. Moloney, "Self-healing femtosecond light filaments," Opt. Lett. 29, 590-592 (2004).

69. F. Courvoisier, V. Boutou, J. Kasparian, E. Salmon, G. Méjean, J. Yu, and J.-P. Wolf, "Ultraintense light filaments transmitted through clouds," Appl. Phys. Lett. 83, 213-215 (2003).

70. P. Kazansky, W. Yang, E. Bricchi, and J. Bovatsek, "“Quill” writing with ultrashort light pulses in transparent materials," Appl. Phys. Lett. 90, 151120 (2007).

71. J. Canning, M. Lancry, K. Cook, and A. Weickman, "Anatomy of a femtosecond laser processed silica waveguide [Invited]," Opt. Mater. Express 1, 998 (2011).

72. Y. Bellouard, E. Barthel, A. A. Said, M. Dugan, and P. Bado, "Scanning thermal microscopy and Raman analysis of bulk fused silica exposed to low energy femtosecond laser pulses," Opt. Express 16, 19520-19534 (2008).

73. E. G. Gamaly, S. Judokazis, K. Nishimura, H. Misawa, B. Luther-Davies, L. Hallo, P. Nicolai, and V. T. Tikhonchuk, "Laser-matter interaction in the bulk of a transparent solid: Confined microexplosion and void formation," Phys. Rev. B 73, 214101 (2006).

74. P. N. Sen and M. F. Thorpe, "Phonons in $\mathrm{AX}_{2}$ glasses: From molecular to band-like modes," Phys. Rev. B 15, 40304038 (1977).

75. C. Fiori and R. Devine, "Evidence for a wide continuum of polymorphs in a-SiO 2, , Phys. Rev. B Condens. Matter 33, 2972-2974 (1986).

76. H. Kakiuchida, K. Saito, and A. J. Ikushima, "Refractive Index, Density and Polarizability of Silica Glass with Various Fictive Temperatures," Jpn. J. Appl. Phys. 43, L743 (2004). 

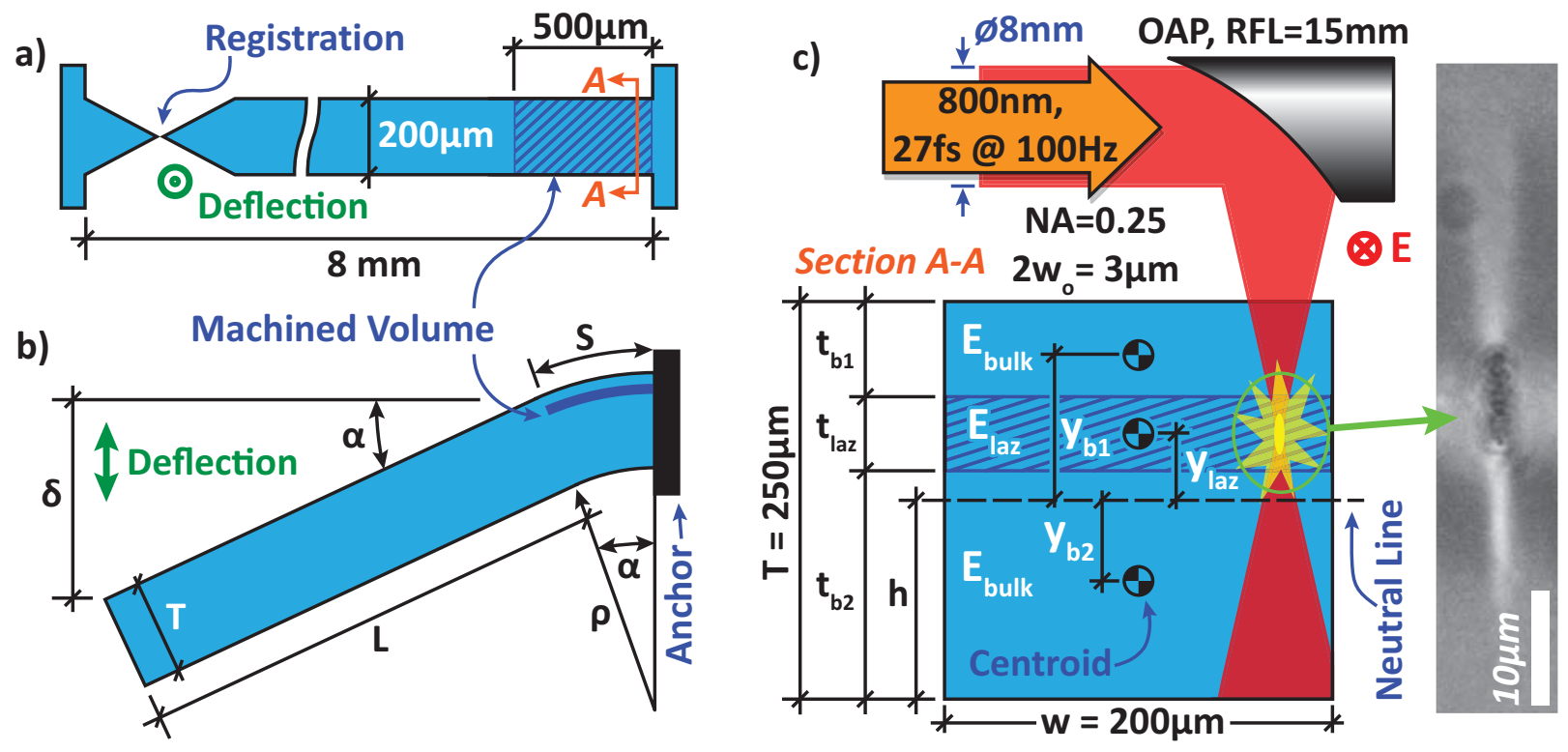

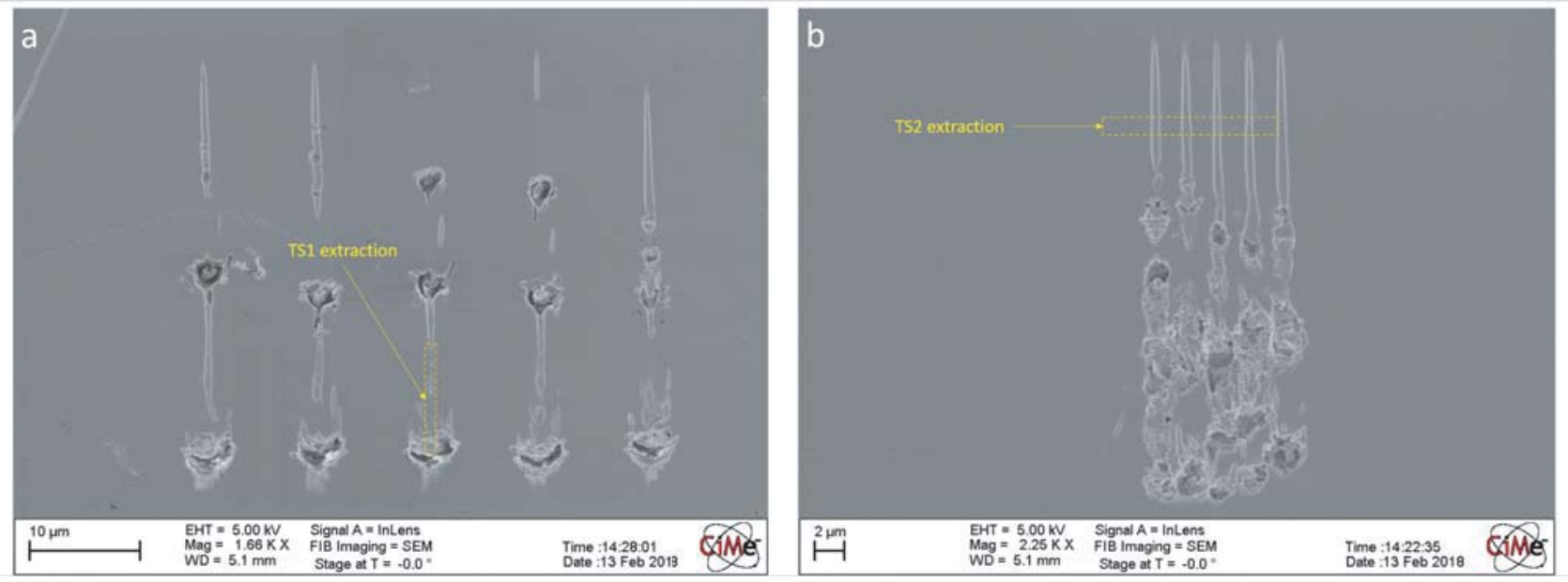


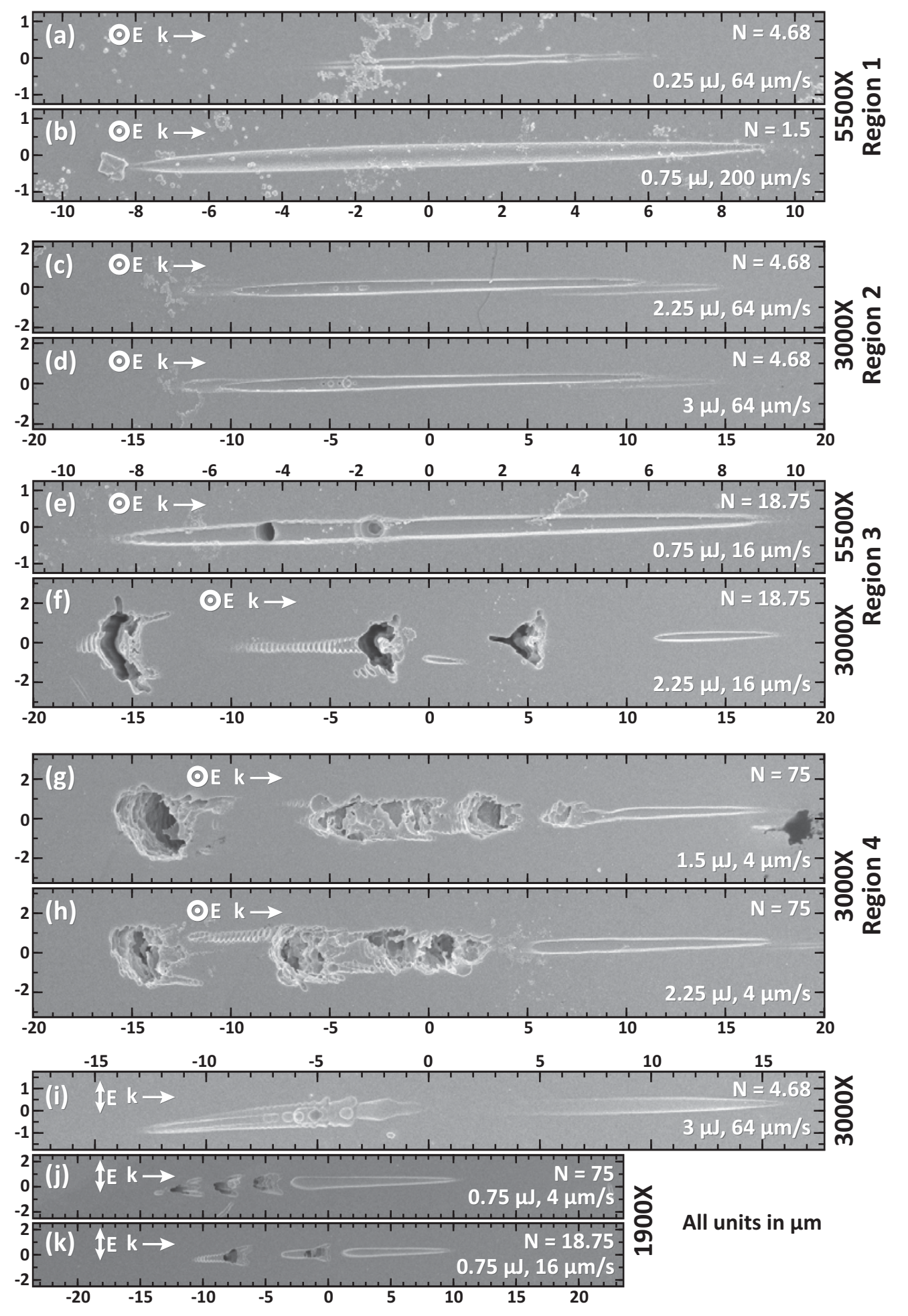




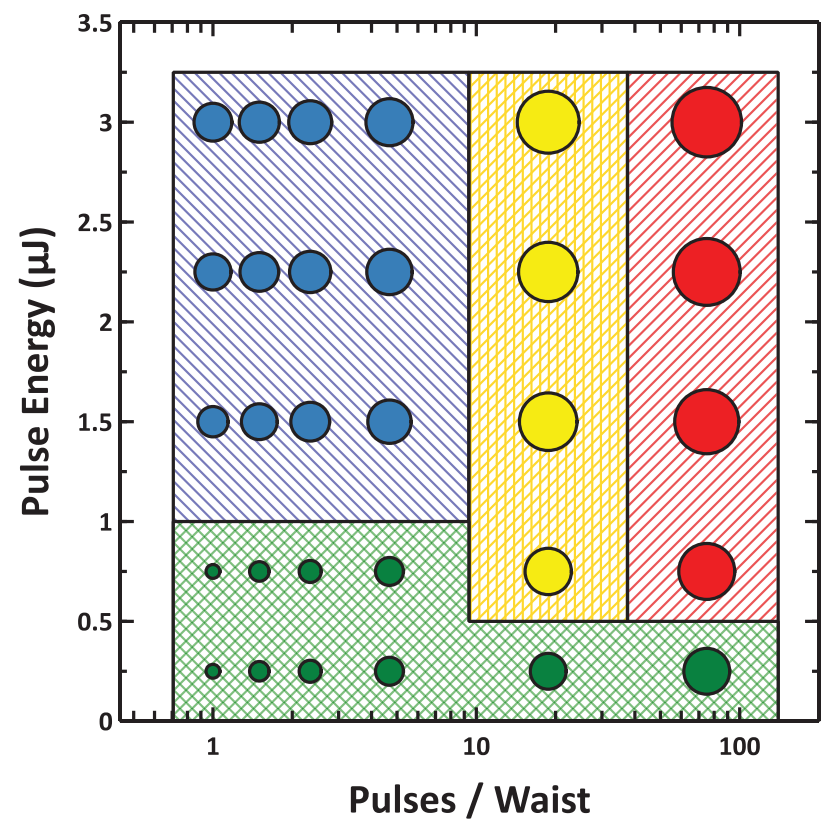




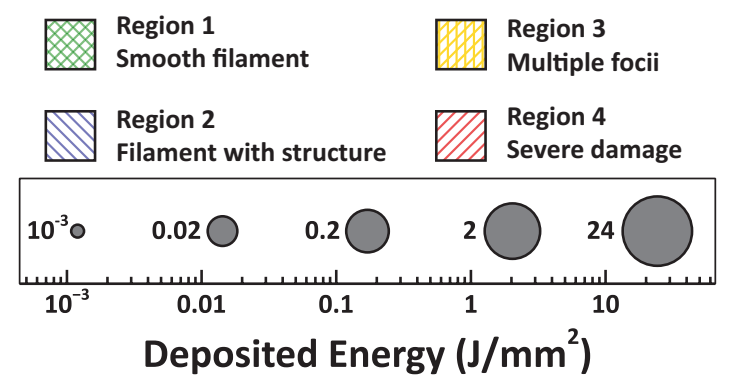




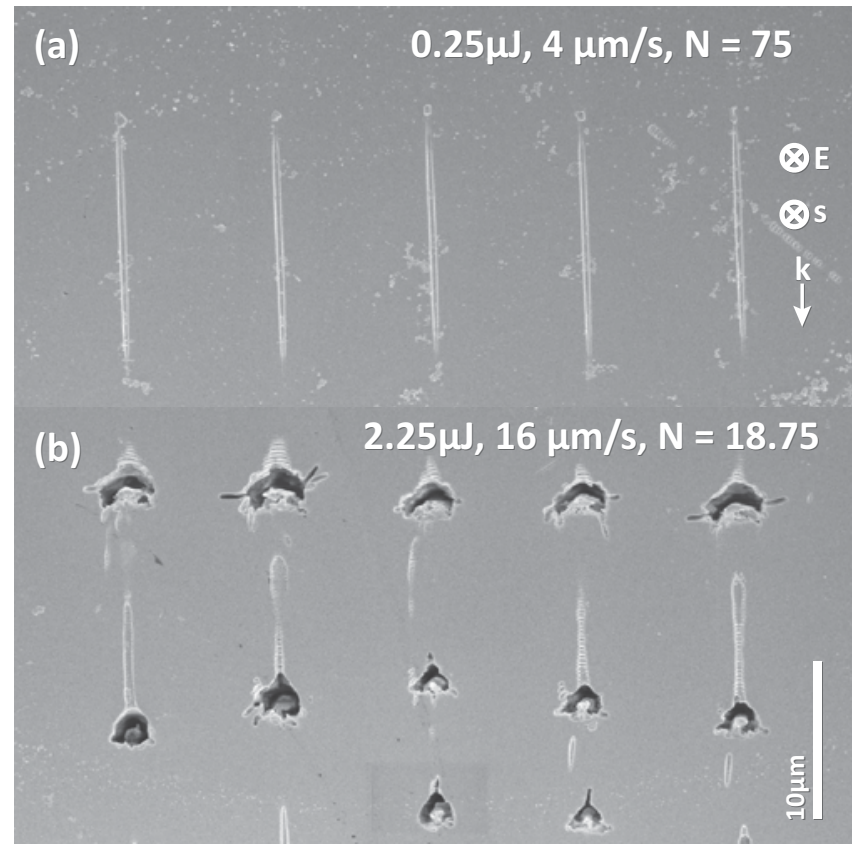




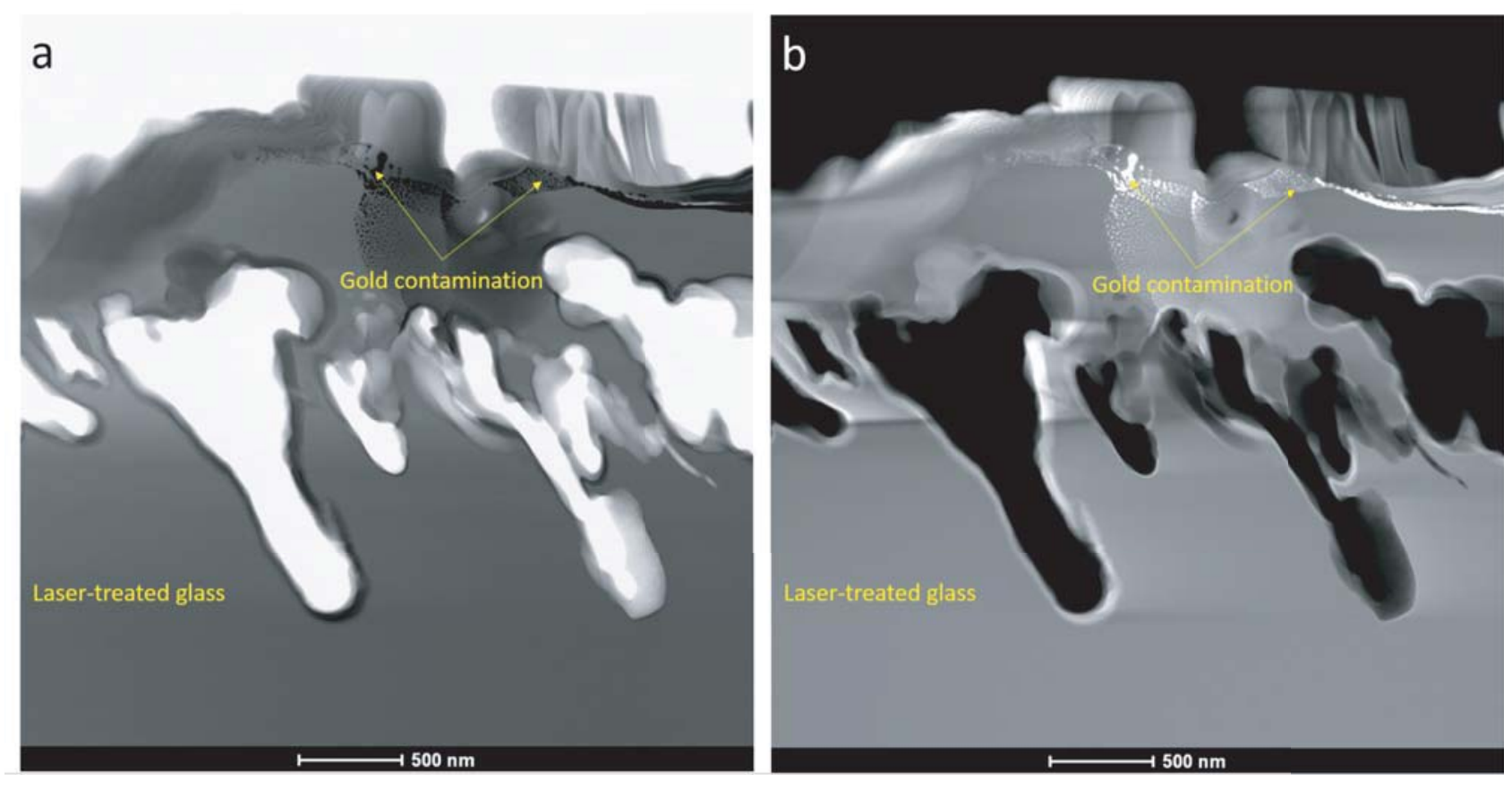





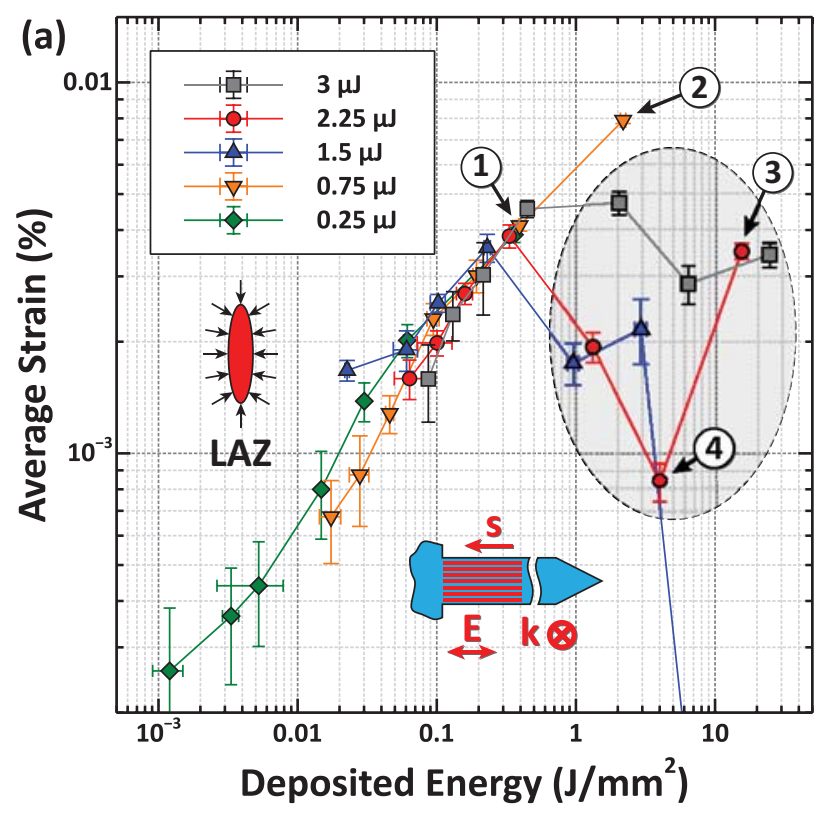




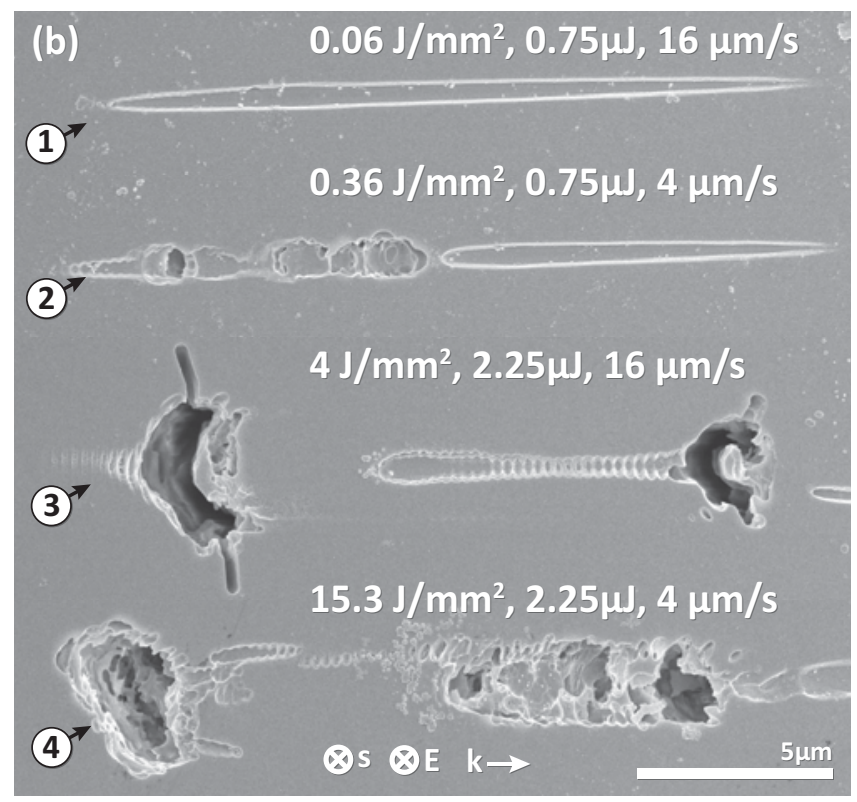




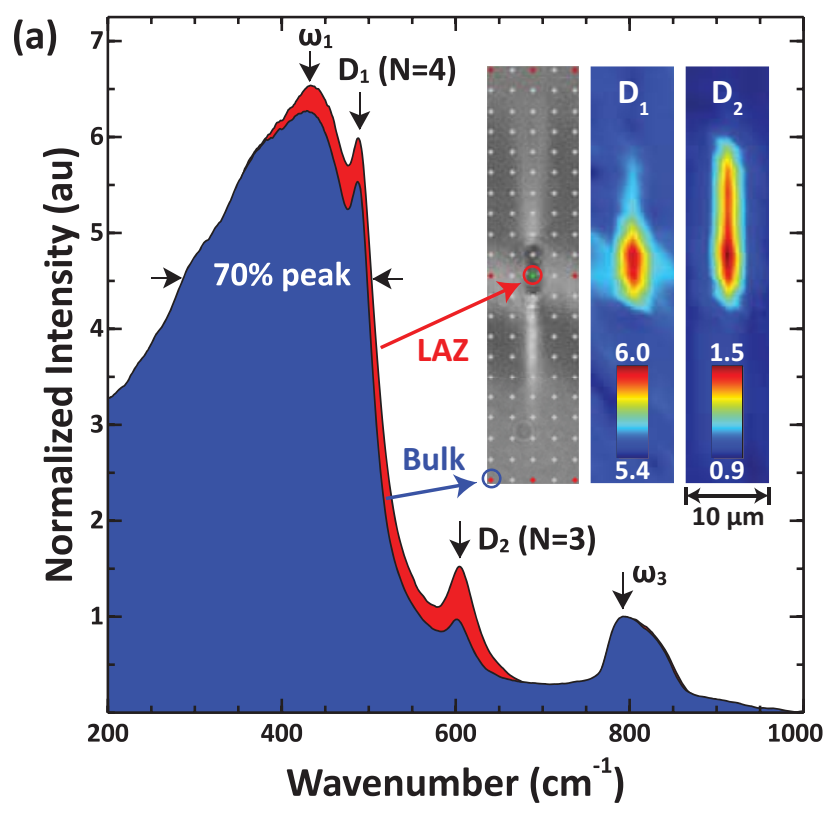




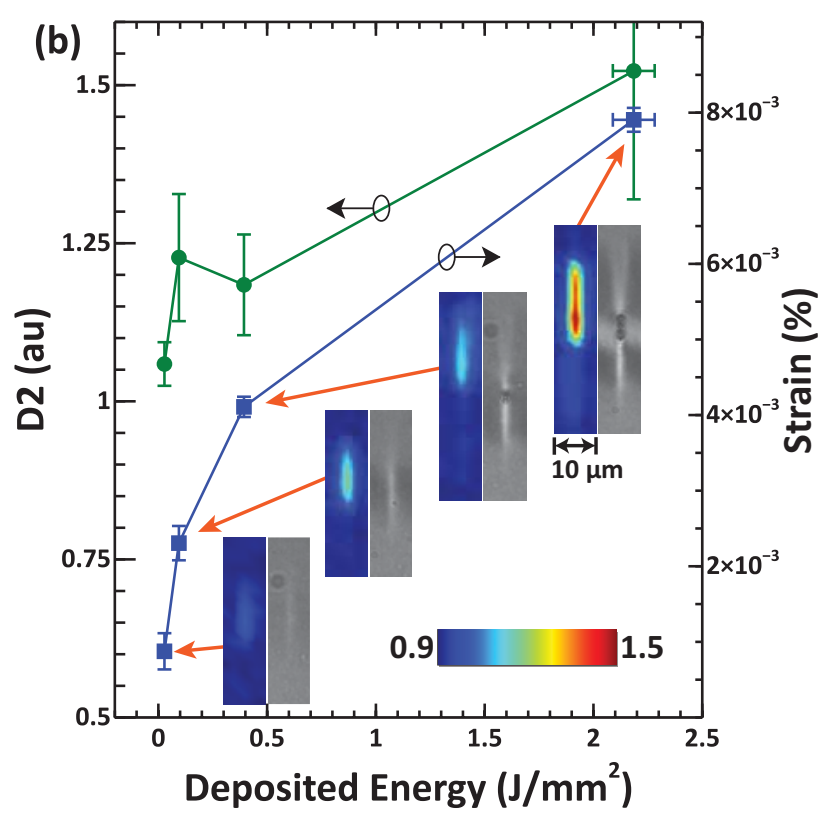




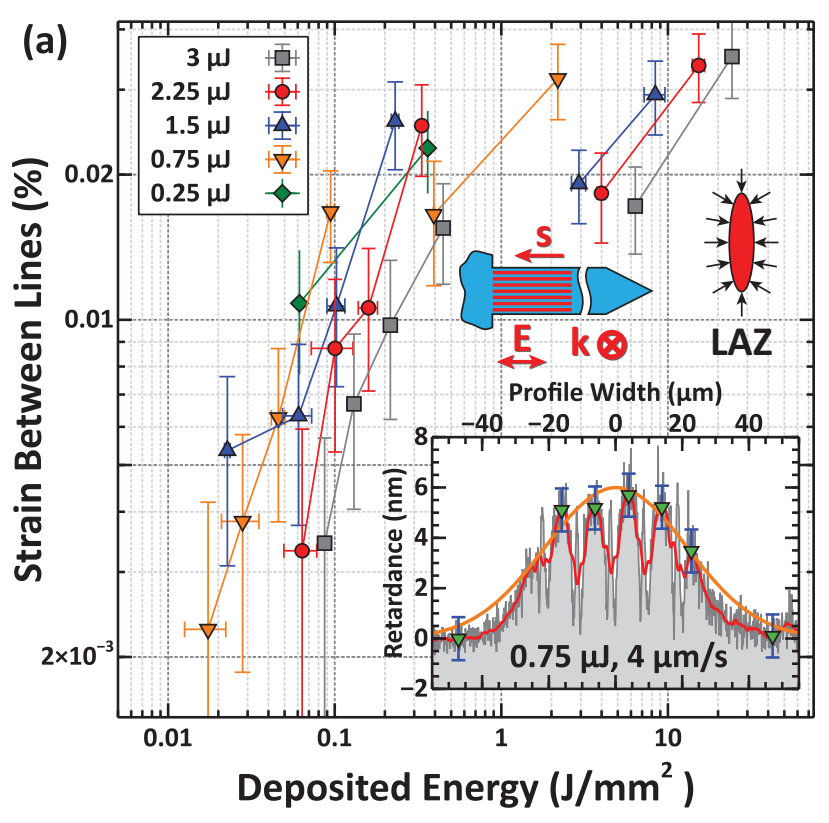




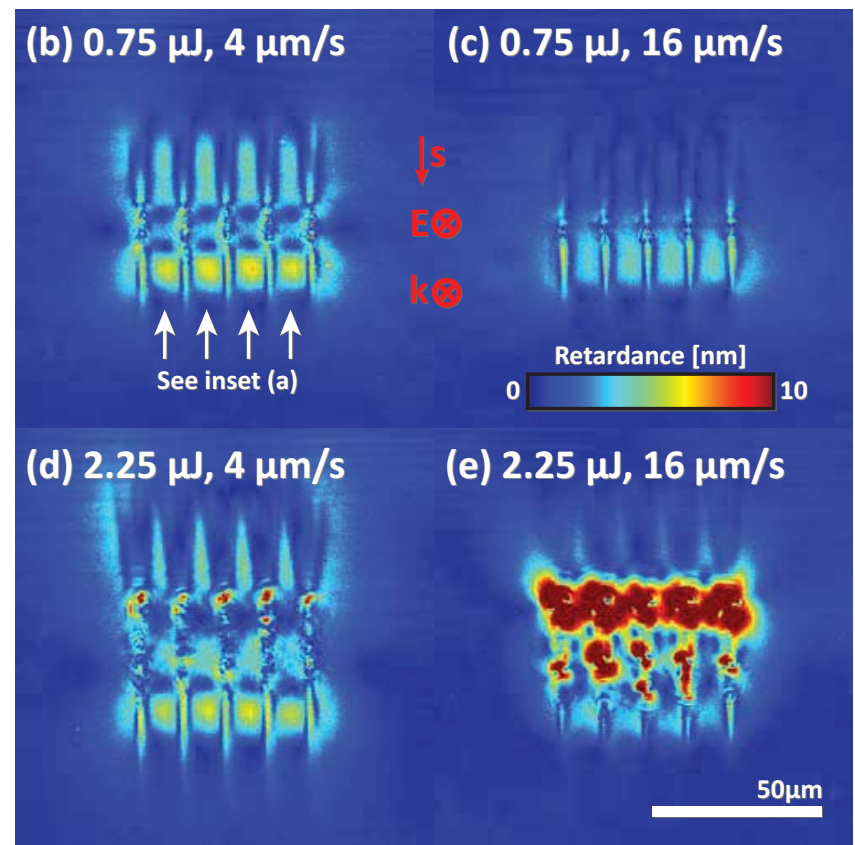




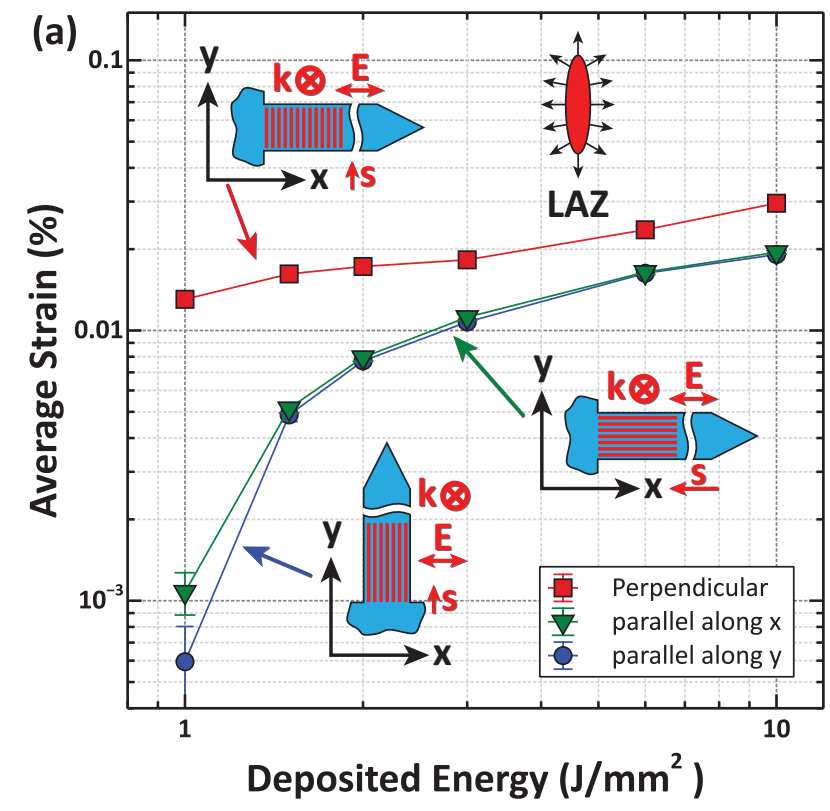

